\title{
Introduction: in vivo cell biology in zebrafish
}

\section{Steffen Scholpp ${ }^{1}$}

Accepted: 3 October 2020 / Published online: 29 October 2020

(c) Springer-Verlag GmbH Germany, part of Springer Nature 2020
Cell biology studies the structure and function of cells- the basic unit of life. Cell architecture, cell communication, cell cycle, and cell metabolism are among many exciting topics of this branch of biology. This special issue "In vivo cell biology in zebrafish" presented by Histochemistry and Cell Biology focuses on the zebrafish as a vital model organism to study many functions of a cell.

In the last decades, cell biology was focussing on culturing cells outside of a body. One major advantage of in vitro cell biology is the possibility to test the response of cells to drugs in order to understand how they function and how the organism would react. One of the most well-known advances using cell culture was probably the development of a vaccine against Polio. At its peak in the 1940s and early 1950s, Polio would paralyze or kill over half a million people worldwide every year. A way to test potential vaccines on a large scale was missing. Using one of the first immortal cell lines, the HeLa cells, a successful vaccine was introduced in the 1950s. Since then, researchers use these traditional two-dimensional monolayer cell cultures, because they are relatively cheap and simple to procure, enabling reliable and efficient cellular assays for high-throughput screening.

However, a significant drawback of these cell culture systems is their failure to capture cellular behaviour within the complexity of an organ system. These simple in vitro models do not account for interactions between different cell types and extracellular matrix in a three-dimensional fashion during development and tissue homeostasis. This may also be reflected in slow and costly drug discovery processes. Currently, many drugs fail in clinical trials due to a lack of understanding of cell behaviour in a complex environment leading to a lack of efficacy or failure due to safety issues. Thus, one of the most promising areas expected to improve our understanding of cell biology - and thus the

Steffen Scholpp

s.scholpp@exeter.ac.uk

1 Living Systems Institute, School of Biosciences, College of Life and Environmental Sciences, University of Exeter, Exeter EX4 4QD, UK success rates in drug development-is the availability of model organisms that better recapitulate in vivo cell biology.

The zebrafish, Danio rerio, is a fast expanding and precious model system for studying many aspects of cell biology within an intact vertebrate organism. Since its introduction as a modern experimental model organism in the early 1980s, many new tools have been developed for imaging and genetic manipulation. Given the high genomic similarities between zebrafish and other vertebrates, many of the critical discoveries in zebrafish are applicable to humans. Zebrafish provide multiple significant advantages over tissue culture models on one hand and mammalian vertebrate model organisms such as mice and rats on the other. Early development of zebrafish embryos is external, rapid, and visually accessible. Zebrafish produce several hundred eggs each week. This high number of offspring is advantageous for genetic mapping studies and large-scale screening experiments in vertebrates. Zebrafish can be raised and maintained in high-density aquarium systems requiring much less space and cost than the animal facilities necessary for mammals. Zebrafish larvae hatch two days after fertilization and begin actively feeding three days after. During these first five days, all major organ systems develop and begin functioning. Techniques including large-scale genome mutagenesis, gene mapping, transgenesis, protein overexpression, gene knockout, cell transplantation for chimeric embryo analysis, and chemical screens have further increased the power of this model organism.

However, the main advantage of this model system is the accessibility of the organism during embryogenesis. Zebrafish embryos and larva are nearly transparent, allowing the scientist to image cells during development-from fertilization to free swimming, actively feeding larval fish. The zebrafish allows us to study organelle function, rearrangement of the cytoskeleton, and complex cell migration processes in the natural environment. In parallel, the development of imaging technologies has significantly enhanced these initial advantages. Zebrafish researchers have capitalized on the optical clarity of the zebrafish embryo by using fluorescent proteins or tagged endogenous fluorescent 
proteins to analyse cells in a living embryo. This method has greatly simplified the analysis of many aspects of cell biology, such as membrane transport, organelle detection, and cell lineage analysis by providing a visual marker in a live organism. The potential of fluorescent proteins and tags in zebrafish is only beginning to be explored. It is now possible to use microscopy techniques in zebrafish that allow such images to have resolutions higher than those imposed by the diffraction limit. Super-resolution microscopy and singlemolecule spectroscopy have been used to quantify single biomolecules and study their behaviour in a living vertebrate organism. In zebrafish, the number of possibilities based on imaging-related techniques has just started to unfold, and novel technologies are continuously being developed and adapted for this model organism.

In this special issue 'In vivo Cell Biology in Zebrafish' we highlight the advantages of the zebrafish as a model for studying cell and developmental processes.

Firstly, Bosze et al. (2020) focus on the functional analysis of the protocadherin 18a. In concert with E-cadherin, Pcdh18a regulate cohort cell migration in the zebrafish notochord primordium. Pinto et al. (2020) review the studies on actin-a major cytoskeletal protein with essential functions in the cell. The article highlights the advantages of zebrafish as an in vivo model for studying actin function during early development, microridge formation, and how to perturb the actin-based cytoskeleton by the usage of small molecule inhibitors. Laurino et al. (2020) describe the effect of blue light on the composition of the zebrafish skin. Dawes et al. (2020) elucidate on measuring protein dynamics in the living zebrafish embryo by spectroscopy techniques. The possibility to measure protein dynamics in an intact organism is exciting and important to allow a veritable analysis of binding characteristics and dynamics of biomolecules in their natural environment.

In a further section, we will discuss the impact of zebrafish studies on regeneration. The function of microRNAs in joint regeneration will be discussed by Lawrence (2020). Measuring strain within cartilage regulates the response of downstream microRNAs. Ryan (2020) describes the importance of adult zebrafish as a model for studying regeneration after heart injury. This article pays particular attention to the cardiac microenvironment in the complex regenerative process.

Finally, the zebrafish has also taken centre stage as a diseases model and in studies on chemical toxicity. Moss et al. (2020) discuss the advantages of the zebrafish as a model for studying protein degradation via autophagy in the context of skeletal development and disease, and the ways these areas are intersecting to facilitate the search for potential therapeutic targets for skeletal disorders. Cook et al. (2020) will discuss the potential of light-sheet microscopy in lysosomal and lipid disorders. Here, zebrafish plays an important role in unravelling the molecular mechanism underlying Niemann-Pick type $\mathrm{C}$ disease and Smith-Lemli-Opitz syndrome. Robinson et al. (2020) show that zebrafish embryos are more sensitive to silver exposure when experiments are initiated at the 1 cell stage, but that pre-exposures do not influence the outcome of subsequent exposures.

In summary, we have collected inspiring examples illustrating the importance of this model organism for singlemolecule studies, state-of-the-art genetic analysis, analysis of complex cohort cell migration, and organ development and regeneration. Thus, the articles of this Special Issue may give the reader a snapshot of how the zebrafish is poised to significantly improve our understanding of the function of a cell in an intact living vertebrate organism.

\section{References}

Bosze B, Ono Y, Mattes B, Sinner C, Gourain V, Thumberger T, Tlili S, Wittbrodt J, Saunders TE, Strähle U, Schug A, Scholpp S (2020) Pcdh18a regulates endocytosis of E-cadherin during axial mesoderm development in zebrafish. Histochem Cell Biol. https://doi. org/10.1007/s00418-020-01887-5

Cook SR, Bladen C, Smith J, Maguire E, Copner J, Fenn GD, Wager K, Waller-Evans W, Lloyd-Evans E (2020) Visualisation of cholesterol and ganglioside GM1 in zebrafish 1 models of NiemannPick type $\mathrm{C}$ disease and Smith-Lemli-Opitz syndrome using light sheet microscopy. Histochem Cell Biol. https://doi.org/10.1007/ s00418-020-01925-2

Dawes M, Soeller C, Scholpp S (2020) Studying molecular interactions in the intact organism-fluorescence correlation spectroscopy in the living zebrafish embryo. Histochem Cell Biol. https://doi. org/10.1007/s00418-020-01930-5

Lauriano ER, Guerrera MC, Laurà R, Capillo G, Pergolizzi S, Aragona M, Abbate F, Germanà A (2020) Effect of light on the calretinin and calbindin expression in skin club cells of adult zebrafish. Histochem Cell Biol. https://doi.org/10.1007/s00418-020-01883-9

Lawrence EA, Hammond CL, Blain EJ (2020) Potential of zebrafish as a model to characterise MicroRNA profiles in mechanically mediated joint degeneration. Histochem Cell Biol. https://doi. org/10.1007/s00418-020-01918-1

Moss JL, Hammond CL, Lane JD (2020) Zebrafish as a model to study autophagy and its role in skeletal development and disease. Histochem Cell Biol. https://doi.org/10.1007/s00418-020-01917-2

Pinto CS, Mishima M, Sampath K (2020) Tools of the trade: studying actin in zebrafish. Histochem Cell Biol. https://doi.org/10.1007/ s00418-020-01932-3

Robinson et al (2020) Developmental exposure window influences silver toxicity but does not affect the susceptibility to subsequent exposures in zebrafish embryos. Histochem Cell Biol. https://doi. org/10.1007/s00418-020-01933-2

Ryan R, Moyse BR, Richardson RJ (2020) Zebrafish cardiac regeneration-looking beyond cardiomyocytes to a complex microenvironment. Histochem Cell Biol. https://doi.org/10.1007/s0041 8-020-01913-6 
News from the Society for Histochemistry

\section{The Robert Feulgen Prize 2020 of the Society for Histochemistry}

has been awarded ex aequo to

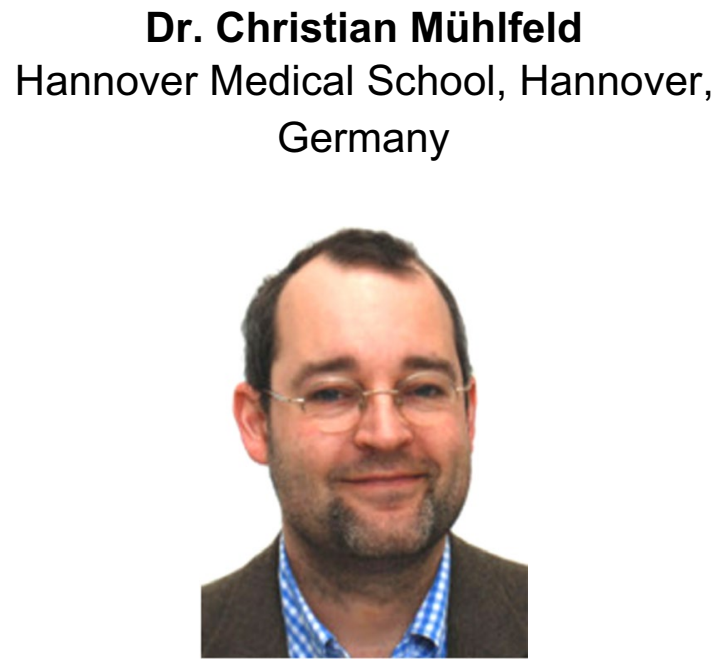

for the development of new stereological and 3D image analysis techniques and their application to the quantitative analysis of the lung alveolar capillary network.

\author{
Dr. Hari Shroff \\ National Institute of Biomedical Imaging \\ and Bioengineering, $\mathrm{NIH}$, \\ Bethesda, MD, USA

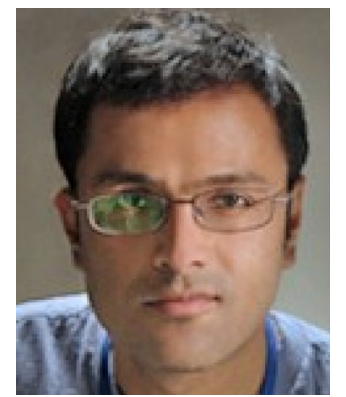 \\ for significant improvement of spatial \\ resolution and speed of image acqui- sition \\ for super-resolution microscopes and their \\ application in the generation of a 4D atlas \\ of neurodevelopment in \\ C. elegans embryos.
}

The Editors of Histochemistry and Cell Biology wish to convey their heartfelt congratulations to Drs. Mühlfeld and Shroff on the receipt of this honor. 


\section{$16^{\text {TH }}$ INTERNATIONAL CONGRESS OF HISTOCHEMISTRY AND CYTOCHEMISTRY}

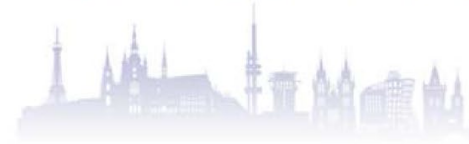

\author{
5 - 8 September \\ PRAGUE 2021
}

Dear Colleagues,

In the light of the COVID-19 pandemic, the ICHC 2020 organizers and IFSHC Executive Council decided to postpone the ICHC 2020 to $\mathbf{5}$ - 8 September 2021. The ICHC 2021 will take place as originally planned in the Cubex Centre, Prague, Czech Republic. The safety of all participants is our top priority. We are sorry for any inconvenience the postponement might have caused you.

The ICHC is held every four years under the auspices of the International Federation of Societies for Histochemistry and Cytochemistry (IFSHC), which continually strives to provide grounds for communication and cooperation among scientists all over the world in the areas of cyto- and histochemistry, cell and tissue biology, microscopy, pathology and other relevant fields.

The city of Prague, also known as the heart of Europe, provides easy access for scientists from all over the world. The congress venue, Cubex Centre Prague which offers technologically and visually unique space, promises to leave everyone with an unforgettable experience. Of course, Prague prides itself with its beautiful historical architecture, technical monuments, celebrated cafés, great food, and beer. This will be underlined by the ICHC gala dinner in the famous Art Nouveau Municipal House, and a free beer party organized in the premises of the Staropramen brewery.

We hope that you will join us in Prague to discuss together your latest achievements and that the venue will provide great opportunities for specialists at all levels of their career, bringing lots of opportunities for strengthening international collaborations. Special attention will be therefore given to the presentations of students. We also expect a rich commercial exhibition where new and emerging technologies will be presented.
We are delighted to inform you that the following speakers will present a lecture at the congress:

Stefan Hell, a Nobel Prize laureate, Max Planck Institute for Biophysical Chemistry, Germany (keynote speaker)

Alev Erisir, Department of Psychology, University of Virginia, USA

Toyoshi Fujimoto, Juntendo University, Nagoya, Japan

Hans-Joachim Gabius, Institute of

Physiological Chemistry, Ludwig Maximilians University of Munich, Germany

Bozena Kamińska, Nencki Institute of Experimental Biology PAS Warszawa, Poland Takehiko Koji, Department of Histology and Cell Biology, Nagasaki University Graduate School of Biomedical Sciences, Nagasaki, Japan Ohad Medalia, Department of Biochemistry, University of Zurich, Switzerland

See you all in Prague, September 2021!

Hinke Multhaupt, President of the IFSHC

Klara Weipoltshammer, President of the Society for Histochemistry

Pavel Hozak, Chair of the Local Organizing Committee

\section{Contacts}

We will keep the current domain: www.ichc2020.com

If you have any questions about registration, please contact: registration@ichc2020.com

If you have any questions about abstracts, please contact: abstracts@ichc2020.com

Other inquiries and comments about the conference, please contact: info@ichc2020.com 


\section{ANNOUNCEMENT}

\section{The Society for Histochemistry}

Invites scientists to apply for the 2021

Robert Feulgen Prize. The prize is awarded for an outstanding achievement in the field of histochemistry.

The contributions may be either towards the development of new histochemical and cytochemical techniques or in the application of existing technology towards solving important problems in biology and/or medicine. Addressed are scientists working in microscopical sciences (in the widest sense) as well as in biochemistry, cell biology, endocrinology, in situ molecular techniques, and neurosciences. Scientists in their mid-career (assistant or associate professor, priv. doz.) are encouraged to apply. The prize is not intended for lifetime contributions.

The Prize consists of a monetary prize of $€ 2,000$

All applications should be submitted before January 31, 2021 via the electronic submission system at: https://www.greception.com/form-login-window/ 191a281d/

The application should contain a short curriculum vitae, a 1,000 word summary of the contributions of the applicant and PDF reprints of the pertinent publications. Full description of conditions is available on the Society website: http://histochemistry.eu/ description_of_conditions_html

Publisher's Note Springer Nature remains neutral with regard to jurisdictional claims in published maps and institutional affiliations. 